\title{
AVALIAÇÃO DE INDICADORES BIOLÓGICOS DE QUALIDADE DO SOLO SOB SISTEMAS DE CULTIVO CONVENCIONAL E ORGÂNICO DE FRUTAS
}

\author{
Evaluation of biological indicators of soil quality under \\ conventional and organic fruit farming system
}

\author{
Deusiane Batista Sampaio1, Ademir Sérgio Ferreira de Araújo², Valdinar Bezerra dos Santos ${ }^{3}$
}

\begin{abstract}
RESUMO
Objetivou-se com este trabalho avaliar os indicadores biológicos de qualidade do solo sob sistemas de cultivo convencional e orgânico de frutas no estado do Piauí. Amostras de solo foram coletadas na projeção da copa das plantas e nas entrelinhas nas profundidades de 0-10, 10-20 e 20-40 cm em duas áreas: sistema orgânico com acerola (SO), sistema convencional com goiaba (SC). Uma área adjacente com vegetação nativa $(\mathrm{AVN})$ foi utilizada como referencial, sendo coletadas amostras de solo nas mesmas profundidades em toda área. As variáveis analisadas foram a respiração basal, carbono orgânico e microbiano e os quocientes respiratório e microbiano do solo. O maior valor de respiração basal foi observado no solo do $\mathrm{SO}_{\text {copa }}$, na profundidade de $10-20 \mathrm{~cm}$. Os solos amostrados na área de vegetação nativa (AVN) e sob a copa das plantas no sistema de cultivo orgânico ( $\mathrm{SO}_{\text {copa }}$ ) apresentaram teores elevados de $C_{\text {org }}$ na superfície $(0-10 \mathrm{~cm})$ e em profundidade $(20-40 \mathrm{~cm})$. Em relação ao solo da AVN, houve aumento no carbono da biomassa microbiana $\left(\mathrm{C}_{\text {mic }}\right)$, nas profundidades de 0-10 cm e 10-20 cm, para o $\mathrm{SO}_{\text {copa }}$ e $\mathrm{SC}_{\text {copa }}$. A adoção do sistema orgânico aumentou a atividade microbiana e o conteúdo de carbono orgânico do solo, mostrando benefícios para esse sistema agrícola.
\end{abstract}

Termos para indexação: Sustentabilidade; microrganismos; atividade microbiana.

\begin{abstract}
The aim of this paper was to evaluate biological indicators of soil quality under conventional and organic farming systems of fruits in Piauí state. Soil samples were collected in the projection area of plant canopies and in the row at a 0-10, 10-20 and 20$40 \mathrm{~cm}$ depths in two areas: organic farming system with "acerola" orchards (SO), conventional farming system with guava orchards (SC). An adjacent area with native vegetation (AVN) was used as referential, being collected soil samples in all area. The variables studied were soil basal respiration, organic and microbial carbon and respiratory and microbial quotients. The higher value of basal respiration was observed in soil of $\mathrm{SO}_{\text {canopies }}$, in $10-20 \mathrm{~cm}$ depth. The soils samples collected in the area of native vegetation (AVN) and under the plant canopies in organic system $\left(\mathrm{SO}_{\text {canopies }}\right)$ showed high organic carbon in the surface $(0-10 \mathrm{~cm}$ depth) and (20-40 cm depth). Compared with soil of AVN, there was an increase in the carbon of microbial biomass $\left(\mathrm{C}_{\text {mic }}\right)$, in 0 $10 \mathrm{~cm}$ and $10-20 \mathrm{~cm}$ depth, for $\mathrm{SO}_{\text {canopies }}$ and $\mathrm{SC}_{\text {canopies }}$. Organic system increased soil microbial activity and organic carbon content, showing benefits for this farming system.
\end{abstract}

Index terms: Sustainability, microorganisms, microbial activity.

(Recebido em 24 de outubro de 2006 e aprovado em 8 de maio de 2007)

\section{INTRODUÇÃO}

O sistema de cultivo convencional apresenta função importante na produção de alimentos, mas é dependente do aporte de insumos químicos, como fertilizantes e pesticidas. Essas práticas agrícolas trazem preocupações em relação ao ambiente e à saúde pública, levando ao aumento do interesse por práticas agrícolas alternativas. Por outro lado, o sistema de cultivo orgânico exclui os aportes de fertilizantes químicos e pesticidas e utilizam técnicas definidas nos programas de certificação (GLOVER et al., 2000). Segundo Mader et al. (2002), em comparação com o sistema de cultivo convencional, o sistema orgânico apresenta o potencial de melhorar a qualidade do solo.

A qualidade do solo é a capacidade dele de funcionar dentro de limites do ecossistema para sustentar a produtividade biológica, manter a qualidade ambiental e promover a saúde vegetal e animal (DORAN \& PARKIN, 1994), podendo ser mensurada através de indicadores biológicos, utilizando microrganismos presentes no solo, pois eles revelam natureza dinâmica e são facilmente afetados por distúrbios causados no solo (KIMPE \& WARKENTIN, 1998).

\footnotetext{
Graduanda em Engenharia Agronômica - Departamento de Agronomia/DAG - Universidade Estadual do Piauí/UESPI - Avenida Nossa Senhora de Fátima - 64202-220 - Parnaiba, PI - deusianeb@yahoo.com.br - Bolsista PIBIC/CNPq/UESPI

'Engenheiro Agrônomo, Doutor - Departamento de Engenharia Agrícola e Solos/DEAS - Universidade Federal do Piaui/UFPI - Campus da Socopo 64000-000 - Teresina, PI - asfaruaj@yahoo.com.br

${ }^{3}$ Engenehiro Agrônomo, Mestre - Departamento de Agronomia/DAG - Universidade Estadual do Piauí/UESPI - Avenida Nossa Senhora de Fátima 64202-220 - Parnaíba, PI - santosvb@bol.com.br
} 
Os principais indicadores biológicos sugeridos para monitorar a qualidade do solo são a biomassa e a atividade microbiana (SPARLING, 1997). A biomassa microbiana é definida como o maior componente vivo da matéria orgânica, compreendendo de 2 a 5\% do carbono orgânico do solo (JENKINSON \& LADD, 1981). Segundo os autores, a biomassa microbiana é um indicador sensível a mudanças nos processo edáficos, em razão da sua taxa de "turnover" ser mais rápida do que do carbono orgânico total do solo.

A respiração basal do solo, avaliada através da liberação de $\mathrm{CO}_{2}$, é a principal medida para avaliar a atividade microbiana do solo (ALEF, 1995), sendo a quantidade de $\mathrm{CO}_{2}$ liberado indicativo do carbono lábil ou prontamente metabolizável do solo (DORAN \& PARKIN, 1994). A respiração do solo é um atributo usado para monitorar a decomposição da matéria orgânica, sendo considerada um importante indicador da atividade microbiana do solo (ANDERSON \& DOMSCH, 1990).

Os indicadores biológicos têm sido freqüentemente usados para avaliar alterações na qualidade do solo pelo uso dos sistemas de plantio direto e convencional (D'ANDREA et al., 2002; VALPASSOS et al., 2001). Nos últimos anos, vários trabalhos foram desenvolvidos avaliando os indicadores biológicos do solo sob sistemas de cultivo convencional e orgânico e os resultados têm mostrado aumentos no conteúdo de matéria orgânica, atividade e biomassa microbiana em solos manejados organicamente (EDMEADES, 2003; GLOVER et al., 2000; MELERO et al., 2005; TU et al., 2006).

Atualmente, considerando a importância dos indicadores biológicos para os processos que ocorrem no solo, verifica-se que estudos a respeito da quantidade e atividade da biomassa microbiana podem fornecer subsídios para o planejamento correto do manejo do solo.

Objetivou-se, aqui, verificar o impacto dos sistemas de cultivo convencional e orgânico de frutas sobre indicadores biológicos de qualidade do solo em um Neossolo Quartzarênico, do estado do Piauí.

\section{MATERIAL E MÉTODOS}

O trabalho foi realizado utilizando-se amostras de solo coletadas no Distrito de Irrigação dos Tabuleiros Litorâneos do Piauí (DITALPI) localizado no município de Parnaíba, norte do estado do Piauí (0305' S; 4147’ W; 46 m). O solo da região é classificado como Neossolo Quartzarênico Órtico Típico, textura arenosa. O clima é do tipo Aw', tropical chuvoso, de acordo com a classificação de Koppen, com precipitação média anual de aproximadamente $1000 \mathrm{~mm}$ e umidade relativa do ar em torno de $75 \%$.

A coleta de solo foi realizada em setembro de 2005 , em três áreas: sistema de cultivo orgânico implantado com a cultura da acerola (SO), sistema convencional de cultivo implantado com a cultura da goiaba (SC), e uma área adjacente com vegetação nativa $(\mathrm{AVN})$ que foi incluída no estudo e utilizada como referencial para as condições naturais do solo. A área de vegetação nativa consiste de uma transição entre Cerrado e Caatinga. O sistema convencional de cultivo vem sendo adotado desde 1999, com culturas anuais e a implantação da cultura da goiaba foi realizada em 2004. As práticas agrícolas do sistema convencional consistiram na correção do solo com $1 \mathrm{t} \mathrm{ha}^{-1}$ de calcário e adubação de implantação com uréia, superfosfato simples, cloreto de potássio e FTE $(30,500,30$ e $50 \mathrm{~g}$ por planta, respectivamente), além de 12 litros planta ${ }^{-1}$ de esterco. A adubação de cobertura consiste da aplicação semestral de uréia, superfosfato simples $(20,500 \mathrm{~g}$ por planta, respectivamente) e 12 litros planta $^{-1}$ de esterco. $\mathrm{O}$ sistema orgânico foi implementado em áreas anteriormente cultivadas sob sistema convencional, e consiste na utilização de práticas definidas nos programas de certificação orgânica. As práticas agrícolas do sistema orgânico iniciaram em 2003 e consistiram no plantio de leguminosas, correção do solo com $1 \mathrm{t} \mathrm{ha}^{-1}$ de calcário e aplicação de $0,5 \mathrm{t} \mathrm{ha}^{-1} \mathrm{de}$ fosfato natural e 1,2 $\mathrm{t} \mathrm{ha}^{-1}$ de MB4. Na implantação da cultura da acerola, em 2004, foram utilizados $500 \mathrm{~kg} \mathrm{ha}^{-1}$ de fosfato natural, $8 \mathrm{~kg}$ planta ${ }^{-1}$ de composto orgânico, $600 \mathrm{~kg} \mathrm{ha}^{-1}$ de MB4 e 100 litros planta ${ }^{-1}$ de palha de carnaúba. Em cobertura semestral são utilizados $600 \mathrm{~kg}$ ha $^{-1}$ de composto orgânico e $8 \mathrm{~kg}_{\text {planta }}{ }^{-1}$ de MB4.

Cada área foi dividida em quatro quadrantes de 0,1 hectares, onde foram coletadas amostras de solo compostas por 10 subamostras simples, por quadrante, nas profundidades de $0-10,10-20$ e $20-40 \mathrm{~cm}$. Os tratamentos consistiram de: a) sistema orgânico com amostragem do solo sob a copa das plantas $\left(\mathrm{SO}_{\text {copa }}\right)$; b) Sistema orgânico com amostragem do solo nas entrelinhas $\left(\mathrm{SO}_{\text {entrelinha }}\right)$; $\left.\mathrm{c}\right)$ sistema convencional com amostragem do solo sob a copa das plantas $\left(\mathrm{SC}_{\text {copa }}\right)$; d) Sistema convencional com amostragem do solo nas entrelinhas $\left(\mathrm{SC}_{\text {entrelinha }}\right)$; e) Área de vegetação nativa $(\mathrm{AVN})$ com amostragem por toda área. As subamostras foram reunidas por profundidade, formando uma amostra composta por quadrante. As amostras foram acondicionadas em sacos plásticos e transportadas em caixa de isopor ao laboratório. As amostras foram peneiradas $(2 \mathrm{~mm})$ e a umidade ajustada 
para $60 \%$ da capacidade de campo, sendo acondicionadas em sacos plásticos com suspiros e mantidas a $4{ }^{\circ} \mathrm{C}$ até a realização das análises.

O teor de carbono orgânico total do solo, nas profundidades de 0-10, 10-20 e 20-40 cm, foi determinado em laboratório conforme Tedesco et al. (1995). As variáveis microbiológicas foram determinadas nas amostras coletadas nas profundidades de 0-10 e 10-20 cm. O carbono da biomassa microbiana foi estimado pelo método da irradiação e incubação (FERREIRA et al., 1999), utilizandose um $\mathrm{K}_{c}$ de 0,45. A taxa de respiração do solo foi estimada pela liberação de $\mathrm{CO}_{2}$ (ALEF, 1995). O quociente microbiano foi calculado pela razão entre o carbono da biomassa microbiana e o carbono orgânico do solo (SPARLING, 1997) e o quociente respiratório $\left(q \mathrm{CO}_{2}\right)$ foi calculado pela razão entre a taxa de respiração e o carbono da biomassa microbiana do solo (ANDERSON \& DOMSCH, 1989).

O delineamento experimental foi inteiramente casualizado com quatro repetições e os resultados foram submetidos à analise de variância e as médias das variáveis em cada profundidade foram comparadas pelo teste de Duncan, a $5 \%$ de probabilidade.

\section{RESULTADOS E DISCUSSÃO}

Houve diferenças significativas nos teores de carbono orgânico $\left(\mathrm{C}_{\mathrm{org}}\right)$ entre os sistemas de cultivo em todas as profundidades avaliadas (Figura 1). Os teores de $\mathrm{C}_{\text {org }}$ nos solos amostrados na área de vegetação nativa (AVN) e sob a copa das plantas no sistema de cultivo orgânico $\left(\mathrm{SO}_{\text {copa }}\right)$, na superfície $(0-10 \mathrm{~cm})$ e em profundidade $(20-40 \mathrm{~cm})$, foram significativamente superiores aos demais solos amostrados. Em subsuperfície $(10-20 \mathrm{~cm})$ não houve diferenças nos teores de $\mathrm{C}_{\text {org }}$ entre os sistemas, exceto para o solo amostrado sob a copa das plantas, no sistema de cultivo convencional $\left(\mathrm{SC}_{\text {copa }}\right.$ ), que apresentou o menor teor de $\mathrm{C}_{\text {org }}$. Considerando o solo da AVN como referencia, observou-se aumento nos teores de $\mathrm{C}_{\text {org }}$ da ordem de $20 \%$ para o solo do $\mathrm{SO}_{\text {copa }}$, nas profundidades de $10-20 \mathrm{~cm}$ e 20 $40 \mathrm{~cm}$, e para os solos do $\mathrm{SC}_{\text {copa }} \mathrm{e} \mathrm{SC}_{\text {entrelinha }}$ houve decréscimo de $40 \%$, em média.

Os valores de $\mathrm{C}_{\text {org }}$, apresentados no $\mathrm{SO}_{\text {copa }}$, indicam que a adição constante e permanente de resíduos orgânicos (composto orgânico e palha), no solo sob a copa das plantas proporcionaram aumento nos teores de $\mathrm{C}_{\text {org }}$, principalmente em profundidade $(10-20 \mathrm{~cm}$ e $20-40 \mathrm{~cm})$.

Segundo Doran (1980), os resíduos orgânicos depositados no solo, após a decomposição, são essenciais no processo de adição de carbono orgânico. Ao longo do tempo, o aumento de carbono orgânico é importante para a sustentabilidade do sistema por causa da influência da matéria orgânica nas propriedades físicas, químicas e biológicas do solo (SPARLING, 1997). Esses resultados são semelhantes aos encontrados por Glover et al. (2000), em sistemas de produção de maçãs, que observaram, após sucessivas aplicações de compostos, aumentos nos teores de $\mathrm{C}_{\text {org }}$ do solo, após quatro anos de adoção do sistema de cultivo orgânico em relação ao sistema convencional.

$0-10 \mathrm{~cm}$

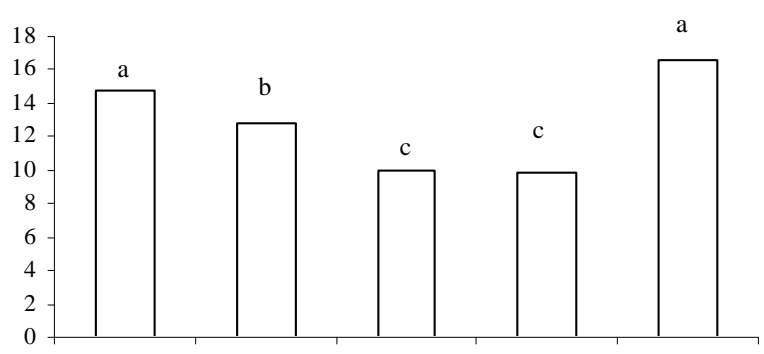

$10-20 \mathrm{~cm}$

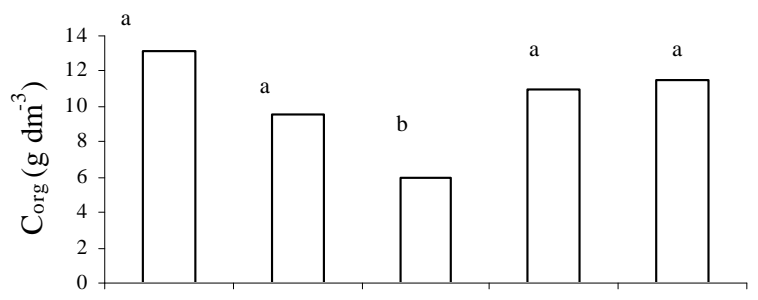

$20-40 \mathrm{~cm}$

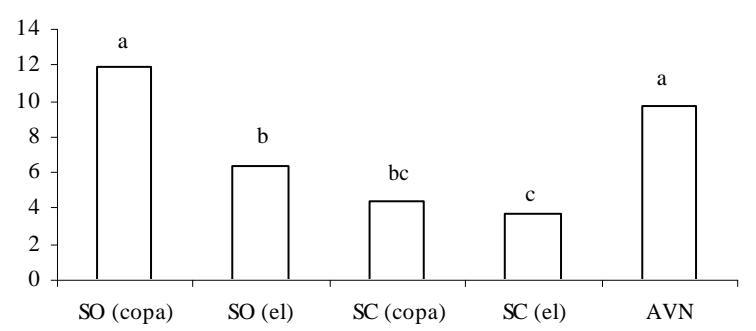

Figura 1 - Carbono orgânico $\left(\mathrm{C}_{\text {org }}\right)$ em Neossolo Quartzarênico, sob sistema de cultivo convencional e orgânico. SO (copa): sistema orgânico com amostragem na projeção da copa das plantas; SO (el): sistema orgânico com amostragem nas entrelinhas; SC (copa): sistema convencional com amostragem na projeção da copa das plantas; SO (el): sistema convencional com amostragem nas entrelinhas; AVN: área de vegetação nativa. 
Em relação ao solo da $\mathrm{AVN}$, houve um aumento no carbono da biomassa microbiana $\left(\mathrm{C}_{\text {mic }}\right)$, nas profundidades de 0-10 cm e $10-20 \mathrm{~cm}$, para os solos amostrados no $\mathrm{SO}_{\text {copa }}$ e $\mathrm{SC}_{\text {copa }}$ (Tabela 1). Além disso, observaram-se diferenças significativas, nas duas profundidades, entre os solos amostrados sob a copa das plantas e nas entrelinhas nos dois sistemas de cultivo. Isso evidencia que o manejo diferenciado do solo, sob a copa das plantas, proporcionou aumento na biomassa microbiana, uma vez que os insumos agrícolas, orgânicos ou minerais, são aplicados nesses locais. Segundo Cattelan \& Vidor (1990), os adubos orgânicos ou minerais contribuem para o crescimento da microbiota do solo, pela disponibilidade de nutrientes às células microbianas.

No solo sob sistema convencional $\left(\mathrm{SC}_{\text {copa }}\right)$, o conteúdo de $\mathrm{C}$ orgânico tendeu a diminuir e não necessariamente levou à redução do $\mathrm{C}$ microbiano, conforme observado por Marchiori Júnior \& Melo (2000), em Latossolo Roxo, sob diferentes manejos. Wardle (1992) cita que os valores de $\mathrm{C}$ microbiano nem sempre se relacionam com o $\mathrm{C}$ orgânico do solo.

Os solos amostrados no $\mathrm{SO}_{\text {copa }}$ e $\mathrm{SC}_{\text {copa }}$, na profundidade de $0-10 \mathrm{~cm}$, apresentaram maior valor de quociente microbiano $\left(\mathrm{C}_{\text {mic }} / \mathrm{C}_{\text {org }}\right)$, comparado com os demais solos (Tabela 1). Enquanto que, na profundidade de $10-20 \mathrm{~cm}$, o maior quociente microbiano foi observado para o solo do $\mathrm{SC}_{\text {copa }}$. Embora sem significância, o menor valor do $\mathrm{C}_{\text {mic }} / \mathrm{C}_{\text {org }}$, na profundidade de $0-10 \mathrm{~cm}$, foi observado no solo da AVN.

Esse maior valor do quociente microbiano, apresentado pelo $\mathrm{SC}_{\text {copa }}$ pode ser em decorrência do menor teor de $\mathrm{C}$ orgânico, observado no solo sob este sistema. Por outro lado, no caso do $\mathrm{SO}_{\text {copa }}$ o maior quociente microbiano sugere que o $\mathrm{C}$ orgânico encontra-se disponível para a microbiota do solo, uma vez que a relação $\mathrm{C}_{\text {mic }} / \mathrm{C}_{\text {org }}$ é um indicador de disponibilidade da matéria orgânica para os microrganismos (ANDERSON \& DOMSCH, 1989), e um alto quociente microbiano indica uma matéria orgânica muito ativa e sujeita a transformações (HART et al., 1989).

O solo do $\mathrm{SO}_{\text {copa }}$, na profundidade de $10-20 \mathrm{~cm}$, apresentou maior respiração basal (liberação de $\mathrm{CO}_{2}$ ) que o demais solos (Tabela 2), indicando maior atividade da biomassa microbiana nesse sistema. Essa maior atividade biológica deve-se à aplicação constante de compostos orgânicos e palhada, que proporciona a presença de matéria orgânica com grandes quantidades de carbono prontamente disponível (SAFFIGNA et al., 1989). Alta atividade microbiana é uma característica desejável, uma vez que pode significar rápida transformação de resíduos orgânicos em nutrientes para as plantas (TU et al., 2006). Resultados semelhantes foram encontrados por Bettiol et al. (2002), que avaliaram a microbiota do solo em sistemas convencional e orgânico e observaram maior atividade microbiana no solo sob sistema orgânico. Os solos da AVN

Tabela 1 - Carbono da biomassa microbiana $\left(\mathrm{C}_{\text {mic }}\right)$ e quociente microbiano $\left(\mathrm{C}_{\text {mic }} / \mathrm{C}_{\text {org }}\right)$ em Neossolo Quartzarênico sob sistema de cultivo convencional e orgânico, em duas profundidades ${ }^{(1)}$.

\begin{tabular}{|c|c|c|}
\hline \multirow[t]{2}{*}{ Sistema de cultivo $^{(2)}$} & \multicolumn{2}{|c|}{ Profundidade $(\mathrm{cm})$} \\
\hline & $0-10$ & $10-20$ \\
\hline & \multicolumn{2}{|c|}{$\mathrm{C}_{\text {mic }}\left(\mathrm{mg} \mathrm{kg}^{-1}\right.$ de solo $)$} \\
\hline SOcopa & $136 \mathrm{a}$ & $137 \mathrm{a}$ \\
\hline SOentrelinha & $82 \mathrm{~b}$ & $43 \mathrm{~b}$ \\
\hline SCcopa & $130 \mathrm{a}$ & $132 \mathrm{a}$ \\
\hline SCentrelinha & $74 \mathrm{~b}$ & $45 \mathrm{~b}$ \\
\hline \multirow[t]{2}{*}{ AVN } & $64 \mathrm{~b}$ & $60 \mathrm{~b}$ \\
\hline & \multicolumn{2}{|c|}{$\mathrm{C}_{\text {mic }} / \mathrm{C}_{\text {org }}(\%)$} \\
\hline SOcopa & $1,08 \mathrm{a}$ & $1,03 \mathrm{~b}$ \\
\hline SOentrelinha & $0,64 \mathrm{bc}$ & $0,44 \mathrm{~b}$ \\
\hline SCcopa & $1,34 \mathrm{a}$ & $2,09 \mathrm{a}$ \\
\hline SCentrelinha & $0,75 \mathrm{~b}$ & $0,48 \mathrm{~b}$ \\
\hline AVN & $0,39 \mathrm{c}$ & $0,53 \mathrm{~b}$ \\
\hline
\end{tabular}


Tabela 2 - Respiração basal e quociente respiratório $\left(q \mathrm{CO}_{2}\right)$ em Neossolo Quartzarênico, sob sistema de cultivo convencional e orgânico, em duas profundidades ${ }^{(1)}$.

\begin{tabular}{lcc}
\hline \multicolumn{1}{c}{ Sistema de cultivo } & \multicolumn{2}{c}{ Profundidade $(\mathrm{cm})$} \\
\cline { 2 - 3 } & $0-10$ & $10-20$ \\
\hline SOcopa & \multicolumn{2}{c}{ Respiração basal $\left(\mathrm{mg} \mathrm{CO}_{2} \mathrm{~kg}^{-1} \mathrm{dia}^{-1}\right)$} \\
SOentrelinha & $105,00 \mathrm{a}$ & $106,70 \mathrm{a}$ \\
SCcopa & $96,43 \mathrm{~b}$ & $91,23 \mathrm{~b}$ \\
SCentrelinha & $100,10 \mathrm{ab}$ & $90,23 \mathrm{~b}$ \\
AVN & $102,30 \mathrm{a}$ & $85,56 \mathrm{~b}$ \\
& $95,83 \mathrm{~b}$ & $85,00 \mathrm{~b}$ \\
SOcopa & & $q \mathrm{CO}_{2}\left(\mathrm{~g} \mathrm{C}^{-C_{2}} \mathrm{~g}^{-1} \mathrm{C}_{\text {mic }} \mathrm{d}^{-1}\right)$ \\
SOentrelinha & $0,78 \mathrm{~b}$ & $0,76 \mathrm{~b}$ \\
SCcopa & $1,16 \mathrm{ab}$ & $2,20 \mathrm{a}$ \\
SCentrelinha & $0,76 \mathrm{~b}$ & $0,67 \mathrm{~b}$ \\
AVN & $1,53 \mathrm{a}$ & $2,03 \mathrm{a}$ \\
\hline
\end{tabular}

(1) Para cada variável, médias seguidas pela mesma letra, na coluna, não diferem entre si pelo teste de Duncan, a 5\% de probabilidade.

(2) SO (copa): sistema orgânico com amostragem na projeção da copa das plantas; SO (entrelinha): sistema orgânico com amostragem nas entrelinhas; SC (copa): sistema convencional com amostragem na projeção da copa das plantas; SO (entrelinha): sistema convencional com amostragem nas entrelinhas; AVN: área de vegetação nativa.

apresentaram os menores valores da respiração basal que os demais solos, mas sem diferenças estatísticas, sugerindo uma maior estabilidade da mata nativa, pois segundo Balota et al. (1998) a baixa respiração basal é um indicativo de ecossistemas mais estáveis.

$\mathrm{O} q \mathrm{CO}_{2}$ indica a eficiência da biomassa microbiana em utilizar o carbono disponível para biossíntese (SAVIOZZI et al., 2002). Os menores valores de quociente respiratório $\left(q \mathrm{CO}_{2}\right)$ foram observados para os solos do $\mathrm{SO}_{\text {copa }}$ e $\mathrm{SC}_{\text {copa }}$, nas duas profundidade (Tabela 2), indicando que, nesses solos, a biomassa microbiana é mais eficiente no uso do $\mathrm{C}$, diminuindo as perdas por $\mathrm{CO}_{2} \mathrm{e}$ incorporando mais tecidos microbianos.

Por outro lado, na profundidade de $10-20 \mathrm{~cm}$, os maiores valores de $q \mathrm{CO}_{2}$ foram observados nos solos amostrados nas entrelinhas nos dois sistemas $\left(\mathrm{SO}_{\text {entrelinha }} \mathrm{e}\right.$ $\mathrm{SC}_{\text {entrelinha }}$ ) e mostra uma menor comunidade microbiana apresentando altas taxas de respiração. Esse resultado reforça que a ausência de insumos agrícolas, orgânicos ou minerais, nas entrelinhas, proporcionou uma menor biomassa microbiana, aumentando o quociente respiratório, uma vez que um alto $q \mathrm{CO}_{2}$ pode ocorrer em razão de uma menor disponibilidade de nutrientes para a microbiota do solo (AGNELLI et al., 2001). Segundo Anderson (1994), o $q \mathrm{CO}_{2}$ fornece uma medida da atividade metabólica, que varia de acordo com o estado da microbiota do solo, a disponibilidade de nutrientes e vários fatores abióticos.

\section{CONCLUSÕES}

A adoção do sistema orgânico aumentou a atividade microbiana e o conteúdo de carbono orgânico do solo, mostrando benefícios para esse sistema agrícola. As práticas agrícolas localizadas, nos sistemas orgânico e convencional, favorecem o crescimento da biomassa microbiana.

\section{AGRADECIMENTOS}

A Fundação de Amparo a Pesquisa no Estado do Piauí (FAPEPI) pelo auxílio financeiro a pesquisa através do Edital PPP2006 (FAPEPI/CNPq).

\section{REFERÊNCIAS BIBLIOGRÁFICAS}

ALEF, K. Estimation of soil respiration. In: ALEF, K.; NANNIPIERI, P. (Eds.). Methods in soil microbiology and biochemistry. New York: Academic, 1995. p. 464-470.

AGNElli, A.; UGOLINI, F. C.; CORTI, G.; PIETRAMELLARA, G. Microbial biomass $\mathrm{C}$ and basal respiration of fine earth and higly altered rock fragments of two Forest soil. Soil Biology \& Biochemistry, Elmsford, v. 33, p. 613-620, 2001. 
ANDERSON, T. H. Physiological analysis of microbial communities in soil: applications and limitations. In: RITZ, K.; DIGHTON, J.; GILLER, K. E. (Eds.). Beyond the biomass. Wiley-Sayce: BSSS, 1994. p. 67-76.

ANDERSON, J. M.; DOMSCH, K. H. Application of ecophysiological quotients ( $\mathrm{qCO}$ and $\mathrm{qD}$ ) on microbial biomass from soils of different cropping histories. Soil Biology \& Biochemistry, Elmsford, v. 22, p. 251-255, 1990

ANDERSON, T. H.; DOMSCH, K. H. Ratio of microbial biomass carbon to total organic carbon in arable soils. Soil Biology \& Biochemistry, Elmsford, v. 21, p. 471-479, 1989.

BALOTA, E. L.; COLOZZI-FILHO, A.; ANDRADE, D. S.; HUNGRIA, M. Biomassa microbiana e sua atividade em solos sob diferentes sistemas de preparo e sucessão de culturas. Revista Brasileira de Ciência do Solo, Campinas, v. 22, p. 641-650, 1998.

BETTIOL, W.; GHINI, R.; GALVÃO, J. A. H.; LIGO, M. A V.; MINEIRO, J. L. C. Soil organisms in organic and conventional cropping systems. Scientia Agricola, Piracicaba, v. 59, p. 656-572, 2002.

CATTELAN, A. J.; VIDOR, C. Flutuações na biomassa, atividade e população microbiana do solo em função de variações ambientais. Revista Brasileira de Ciência do Solo, Campinas, v. 14, p. 133-142, 1990.

D'ANDREA, A. F. D.; SILVA, M. L. N.; CURI, N.; SIQUEIRA, J. O.; CARNEIRO, M. A. C. Atributos biológicos indicadores da qualidade do solo em sistemas de manejo na região do cerrado no sul do estado de Goiás. Revista Brasileira de Ciência do Solo, Campinas, v. 26, p. 913-923, 2002.

DORAN, J. W. Soil microbial and biochemical changes associated with reduced tillage. Soil Science Society American Journal, Madison, v. 44, p. 765-771, 1980.

DORAN, J. W.; PARKIN, T. B. Defining and assessing soil quality. In: DORAN, J. W.; COLEMAN, D. C.; BEZDICEK, D. F.; STEWART, B. A. (Eds.). Defining soil quality for a sustainable environment. Madison: SSSA, 1994. p. 3-21.

EDMEADES, D. C. The long-term effects of manures and fertilisers on soil productiviy and quality: a review. Nutrients Cycling in Agroecosystem, [S.1.], v. 66, p. 165180, 2003.
FERREIRA, A. S.; CAMARGO, F. A. O.; VIDOR, C. Utilização de microondas na avaliação da biomassa microbiana do solo. Revista Brasileira de Ciências do Solo, Campinas, v. 23, p. 991-996, 1999.

GLOVER, J. D.; REGANOLD, J. P.; ANDREWS, P. K. Systematic method for rating soil quality of convencional, organic, and integrated apple orchards in Washington State. Agriculture, Ecosystem \& Environment, [S.1.], v. 80, p. 29-45, 2000.

HART, P. B. S.; AUGUST, J. A.; WEST, A. W. Long-term consequences of topsoil mining on select biological and physical characteristics of two New Zealand loessial soils under grazed pasture. Land Degradation, [S.1.], v. 1, p. 7788, 1989

JENKINSON, D. S.; LADD, J. N. Microbial biomass in soil: measurement and turnover. In: PAUL, E. A.; LADD, J. N. (Eds.). Soil biochemistry. New York: M. Dekker, 1981. p. 415-471

KIMPE, C. R.; WARKENTIN, B. P. Soil functions and the future of natural resources. Advances in GeoEcology, [S.1.], v. 31, p. 3-10, 1998.

MÄDER, P.; FLIEBACH, A.; DUBOIS, D.; GUNST, L.; FRIED, P.; NIGGLI, U. Soil fertility and biodiversity in organic farming. Science, London, v. 296, p. 1694-1697, 2002.

MARCHIORI JÚNIOR, M.; MELO, W. J. Alterações na matéria orgânica e na biomassa microbiana em solo de mata natural submetido a diferentes manejos. Pesquisa Agropecuária Brasileira, Brasília, v. 35, p. 1177-1182, 2000.

MELERO, S.; PORRAS, J. C. R.; HERENCIA, J. F.; MADEJON, E. Chemical and biochemical properties in a silty loam soil under conventional and organic management. Soil \& Tillage Research, Amsterdam, v. 90, p. 162-170, 2005.

SAFFIGNA, P. G.; POWLSON, D. S.; BROOKES, P. C.; THOMAS, G. A. Influence of sorghum residues and tillage on soil organic matter and soil microbial biomass in an Autralian vertissol. Soil Biology \& Biochemistry, Elmsford, v. 21, p. 759-765, 1989.

SAVIOZZI, A.; BUFALINO, P.; LEVI-MINZI, R.; RIFFALD, R. Biochemical activities in a degraded soil restored by two amendments: a laboratory study. Biology \& Fertility of Soils, Berlin, v. 35, p. 96-101, 2002. 
SPARLING, G. P. Soil microbial biomass, activity and nutrient cycling as indicators of soil health. In: PANKHURST, C.; DOUBE, B. M.; GUPTA, V. V. S. R. (Eds.). Biological indicators of soil health. Cambridge: $\mathrm{CAB}$ International, 1997. p. $97-120$.

TEDESCO, M. J.; GIANELLO, C.; BISSANI, C. A. Análises de solos, plantas e outros materiais. Porto Alegre: UFRGS, 1995. 174 p. (Boletim técnico, 5).

TU, C.; RISTAINO, J. B.; HU, S. Soil microbial biomass and activity in organic tomato farming systems: effects of organic inputs and straw mulching. Soil Biology \& Biochemistry, Elmsford, v. 38, p. 247-255, 2006.

VALPASSOS, M. A. R.; CAVALCANTE, E. G. S.; CASSIOLATO, A. M.; ALVES, M. C. Effects of soil management systems on soil microbial activity, bulk density and chemical properties. Pesquisa Agropecuária Brasileira, Brasília, v. 36, p. 1539-1545, 2001.

WARDLE, D. A. A comparative assessment of factors with influence of microbial biomass carbon and nitrogen levels in soil. Biological Review, [S.1.], v. 67, p. 321-358, 1992. 